\title{
Disección Aórtica manejo clínico quirúrgico a propósito de 4 \\ casos
}

\author{
Aortic dissection surgical clinical management about 4 cases \\ Mauricio Trelles Correa. ${ }^{1}$, Hanny Yamunaque Vire. ${ }^{2}$, Edisson Luna Castro. ${ }^{3}$ \\ Recibido: 12-09-2019 / Revisado: 20-10-2019 /Aceptado:10-11-2019/ Publicado: 07-12-2019
}

\begin{abstract}
DOI: https://doi.org/10.33262/cienciadigital.v3i4.2.1020

Introduction: Aortic dissection is a medical emergency that is associated with high mortality. It is a medical entity that in most cases in a precise surgical treatment. Methods: Longitudinal descriptive study with retrospective of 4 cases surgically intervened by diagnosis of aortic dissection, between November 2016 and November 2018 attended at Clinical de Epicainides Latino. Results: The average age of the patients was 52.5 years, with a maximum of 70 and a minimum of 20 , all were male, they had a history of hypertension $50 \%$, a chronic pericarditis due to possible tuberculosis, 2 of them are smokers, they were operated on $100 \%$ of the patients, 1 of the cases, the initial diagnosis was made by transesophageal echocardiography and confirmed by angiotomography. the average days of hospitalization was 22 days, an average of 17.5, preoperative Euro score was a maximum of 3.61 and minimum of 0.7 , the type of dissection according to the Stanford scale were type A in all 4 cases, maximum bleeding during the surgery was 1800 and minimum $300 \mathrm{cc}$, the total patients were discharged alive and in stable condition. Conclusions: Clinical research and the experience of the surgeon remain the key factors for the diagnosis and implementation of the appropriate surgical technique, the results can be modified in their natural history and improve prognosis.
\end{abstract}

Keywords: Type A aortic dissection, Reoperation, cardiac surgery.

${ }^{1}$ Clínica de Especialidades Latino, matc2202@hotmail.com

${ }^{2}$ Clínica de Especialidades Latino, hannbad@gmail.com

${ }^{3}$ Clínica de Especialidades Latino, edissonluna_87@hotmail.com 


\section{Resumen}

Introducción: La disección aórtica es una emergencia médica que se asocia a una elevada mortalidad. Se trata de una entidad médica que en la mayoría de los casos va a precisar tratamiento quirúrgico. Método: Estudio descriptivo longitudinal con toma retrospectiva de 4 casos intervenidos quirúrgicamente por diagnóstico de disección aortica, entre noviembre de 2016 y noviembre de 2018 atendidos en la Clínica de Especialidades "Latino". Resultados: La edad promedio de los pacientes fue de 52,5 años, con un máximo 70 y mínimo de 20, todos fueron varones, tenían antecedentes de hipertensión el 50\%, una pericarditis crónica por posible tuberculosis, 2 de ellos son fumadores, fueron intervenidos quirúrgicamente el $100 \%$ de los pacientes, 1 de los casos el diagnóstico inicial fue realizado por ecocardiograma transesofágico y confirmado por angiotomografia. los días promedio de hospitalización fue 22 días, una media de 17,5, Euro score preoperatorio fue un máximo de 3.61 y mínimo de 0.7, el tipo de disección según la escala de Stanford fueron de tipo A los 4 casos, sangrado máximo durante la cirugía fue de 1800 y mínimo 300 CC., el total de pacientes fueron dados de alta vivos y en condición estable. Conclusión: La sospecha clínica y la experiencia del cirujano siguen siendo los factores clave para el diagnóstico y la implementación de la técnica quirúrgica adecuada, los resultados pueden modificar su historia natural y mejorar el pronóstico.

Palabras clave: Disección aortica tipo A, reintervención quirúrgica, cirugía cardiaca.

\section{Introducción}

La disección aórtica aguda es un diagnóstico que puede pasar por alto fácilmente entre los pacientes con dolor torácico agudo, por lo tanto, se requiere obtener una gran sospecha y el uso de imágenes cardiovasculares avanzadas, de modo que se pueda iniciar un tratamiento oportuno. Según la clasificación de Stanford tenemos tipo A y tipo B, en el presente estudio describen cuatro casos de la más común que es el tipo A, la cual suele ser letal con una alta mortalidad y morbilidad $(1,2)$. La edad entre 40 y 70 años es el rango en donde se da aproximadamente el $75 \%$ de las disecciones aórticas, además es tres veces más común en hombres que en mujeres (1).

Los síntomas clínicos comunes en la disección tipo A son el dolor torácico con cualquier hallazgo neurológico, la combinación de dolor torácico y abdominal, o dolor torácico acompañado de 
debilidad en las extremidades o parestesia debe alertar al médico sobre la posibilidad de disección aortica (2).

El tratamiento quirúrgico consiste básicamente en cerrar el puerto de entrada de disección y reemplazar la aorta ascendente y el arco aórtico si es necesario, habiendo reparado y reconstruido la aorta distal de antemano. La técnica proporciona información precisa sobre la ubicación del puerto de entrada, la etiopatogenia de la incompetencia aórtica y los trastornos del arco distal y la aorta torácica descendente $(2,3)$.

Al menos el $30 \%$ de los pacientes mueren después de llegar a la sala de emergencias e incluso después de la cirugía, las tasas de mortalidad varían de 20 a 30\%. Los pacientes que sobreviven a la cirugía, la comorbilidad también tiene un costo y la calidad de vida es pobre. La mortalidad más alta de una disección aórtica aguda está dentro de los primeros 10 días. Los pacientes con una disección crónica tienden a tener un mejor pronóstico, pero incluso su esperanza de vida se acorta en comparación con la población general (4).

\section{Método}

Estudio descriptivo longitudinal con toma retrospectiva de 4 casos intervenidos quirúrgicamente por diagnóstico de disección aortica, entre noviembre de 2016 y noviembre de 2018 atendidos en la Clínica de Especialidades "Latino".

\section{Resultados}

Se realizó la cirugía cardiaca por disección aórtica en cuatro casos pacientes de sexo masculino, que fueron intervenidos quirúrgicamente en la Clínica de Especialidades Latino durante el periodo comprendido entre noviembre de 2016 y noviembre de 2018. En el primer caso sus diagnósticos fueron: Aneurisma de raíz de aorta ascendente, Anuloectasia aórtica, Disección aórtica tipo A según la clasificación de Stanford, Insuficiencia aórtica severa, el diagnóstico se lo realizó por angiotomografía y ecocardiograma transtorácico, como antecedente patológico personal el paciente presentaba hipertensión arterial de larga data bien controlada, el EURO SCORE II fue de 6.88, se realizó la cirugía de Bentall-Bono con tubo valvulado mecánico San Jude No 25 y válvula tipo mecánica , tiempo de circulación extracorpórea: 3horas 23minutos, tiempo de clampeo: 3 horas 55 minutos, sangrado aproximado $400 \mathrm{CC}$., con necesidad de uso de hemoderivados, presentando síndrome de bajo gasto como complicación transoperatoria, en el postquirúrgico permanece en la unidad de cuidados intensivos bajo ventilación mecánica durante 1 día, con necesidad de apoyo vasopresor e inotrópico, los días de hospitalización fueron 12, fue dado de alta vivo en condición 
estable, control a los 30 días estable, sin embargo 3 meses posteriores a la cirugía presentó sepsis, el germen aislado fue Klebsiella multiresistente (KPC).

El segundo caso se trata de un varón de 64 años de edad, no refiere antecedentes patológicos personales, sus diagnósticos fueron: Aneurisma disecante de aorta tipo A según la clasificación de Stanford, Hidronefrosis, Litiasis renal, el diagnóstico se lo realizó mediante angiotomografía y ecocardiograma transtorácico, EURO SCORE II fue de 11.92, se realizó cirugía reemplazo de aorta ascendente por tubo de Dacron, tiempo de circulación extracorpórea: 2horas 30minutos, tiempo de clampeo: 2 horas 17 minutos, sangrado aproximado de $3000 \mathrm{CC}$. se presentó como complicación intraoperatoria sangrado en aortorrafia proximal por lo que egresó de quirófano empaquetado y requirió nuevamente intervención quirúrgica a las 48 horas, una complicación post operatoria fue coagulopatía por lo que se requirió además del uso de hemoderivados, el uso de complejo de protrombina humana a dosis máxima, se mantuvo bajo ventilación mecánica durante 8 días, y 32 días hospitalizado siendo dado de alta en condición estable.

El tercer caso se trata de paciente masculino de 20 años de edad, con antecedente de derrame pleural y pericarditis crónica por posible tuberculosis en tratamiento, como hábitos fuma e ingiere alcohol ocasionalmente, fue ingresado con el diagnóstico de aneurisma de aorta ascendente, disección aortica tipo A según la clasificación de Stanford, el diagnostico se lo realizó con angiotomografia y ecocardiograma transesofágico, paciente previo a la cirugía presenta choque el EUROSCORE II fue de 10.55, se realizó reemplazo de aorta ascendente por prótesis Dacron No 20, tiempo de circulación extracorpórea: 2 horas 15minutos, tiempo de clampeo: 1 hora 40 minutos sangrado aproximado 1800 CC., como complicaciones transoperatorias se presentó sangrado en aortorrafia, fue necesaria la utilización de hemoderivados, en su post quirúrgico permanece bajo ventilación mecánica durante 1 día, total de días de hospitalización 42 días, fue dado de alta en condición estable.

El cuarto paciente es un varón de 70 años de edad, con antecedente de hipertensión arterial de varios años de evolución en tratamiento y bien controlada, hábito de tabaquismo con una media de consumo de 20 paquetes/año, fue ingresado con los diagnósticos de: Aneurisma de raíz de aorta ascendente, anuloectasia aortica, Disección aortica tipo A según clasificación de Stanford, Insuficiencia aórtica severa, Insuficiencia mitral severa el diagnóstico fue realizado con angiotomografía y ecocardiograma transtorácico, EURO SCORE II de 16.96, se realiza cirugía de Bentall-Bono, tubo valvulado mecánico marca San Jude No. 25, válvula tipo mecánica, tiempo de 
circulación extracorpórea: 2horas 47minutos, tiempo de clampeo: 2 horas 35 minutos, sangrado aproximado durante la cirugía 800cc fue necesaria la utilización de hemoderivados, como complicación transoperatoria presentó síndrome de bajo gasto cardíaco, permanece en la unidad de cuidados intensivos bajo ventilación mecánica durante 4 días, total de días de hospitalización 15, fue dado de alta en condición estable control dentro de 30 días sin complicaciones, ecocardiograma de control reporte normal.

La edad promedio fue de 52.5 años, todos fueron varones, tenían antecedentes de hipertensión arterial el 50\%, 1 paciente con pericarditis crónica por posible tuberculosis, 2 de ellos son fumadores. Todos fueron diagnosticados por angiotomografía y ecocardiograma transtorácico, excepto 1 caso con ecocardiograma transesofágico, EURO SCORE II promedio fue de 11.57, los días promedio de hospitalización fue de 22 días, una media de 17.5, el tipo de disección según la escala de Stanford fueron de tipo A los 4 casos, en todos los casos fue necesario el uso de hemoderivados y en 1 de los casos el uso de complejo de protrombina humana a dosis máxima, en todos los casos fue necesario el uso de vasopresores e inotrópicos, las complicaciones transoperatorias fueron el síndrome de bajo gasto cardíaco en 2 casos y sangrado en aortorrafía en 2 casos, de los cuales 1 egresó de quirófano empaquetado y requirió de reintervención quirúrgica en 48 horas, todos fueron dados de alta vivos y en condición estable. Seguimiento a 30 días mortalidad 0. Seguimiento a 1 año; 1 fallecimiento por sepsis, germen aislado Klebsiella multiresistente (KPC) al tercer mes de la cirugía, los restantes control ecocardiográfico normal incluido en 1 paciente además tenía el diagnóstico de insuficiencia mitral severa funcional al momento de la cirugía.

\section{Discusión}

La disección aórtica es una emergencia médica poco frecuente que pone en peligro la vida y se asocia con una alta mortalidad quirúrgica de hasta el $25 \%(1,2)$. Se describen cuatro casos tipo A según la clasificación de Stanford. Entre los factores de riesgo que prevalecen son la hipertensión, edad avanzada y enfermedades de la pared aórtica (7). En un estudio realizado por Rejendra y colaboradores identifico como factor predictivo mayor a setenta años de edad (8). En la clínica el procedimiento diagnóstico más accesible es angiotomografía y ecocardiograma torácico.

La decisión sobre la técnica dependerá de la experiencia del cirujano y de la condición clínica del paciente, se ha implementado la técnica de Bentall - Bono en los cuatro casos descritos. Los avances continuos en las tecnologías de imagen y tratamiento están mejorando, la angiotomografía 
computarizada (AngioTAC) es una herramienta de diagnóstico más confiable, se puede evaluar el desplazamiento interno de la íntima $(3,4,5)$, en este caso todos los casos se diagnosticó por angiotomografía. En un estudio publicado en el año 2015 indicó que el ecocardiograma transtorácico es una herramienta fundamental en el área de emergencia para la evaluación de la sospecha de disección aortica (12). El ecocardiograma transesofágica se puede ser útil para el diagnóstico de patologías aórticas torácicas agudas, detección temprana e intervenciones correctivas inmediatas (3).

Wang y colaboradores en un estudio realizado entre 2005 y 2016 se demostró que el 23.6\% de los pacientes requirió al menos una reintervención quirúrgica después de la cirugía inicial (13), como en el segundo caso ya descrito.

La evolución a medio - largo plazo de los pacientes con disección aórtica tipo A depende de las complicaciones postquirúrgicas que se presentes, la supervivencia a los 5 años de los pacientes dados de alta se un estudio es del 65 - 85\% (13). Según Wang y colaboradores tuvieron como resultado la mortalidad a los 30 días del alta equivalente al 7\% (13), en comparación con el presente estudio no se describe resultados desfavorables a los 30 días postquirúrgicos, sin embargo al año de seguimiento un paciente presento sepsis y posteriormente falleció.

Conclusión. La disección aortica aguda continúa con una alta mortalidad a pesar del progreso diagnóstico y terapéutico durante la última década. Sin embargo, la sospecha clínica y la experiencia del cirujano siguen siendo los factores clave para el diagnóstico y la implementación de la técnica quirúrgica adecuada, los resultados pueden modificar su historia natural y mejorar el pronóstico.

\section{Referencias Bibliográficas}

1. Levy D, Le JK. Aortic Dissection. En: StatPearls [Internet]. Treasure Island (FL): StatPearls Publishing; 2019 [citado 15 de marzo de 2019]. Disponible en: http://www.ncbi.nlm.nih.gov/books/NBK441963/

2. Masip AE, Masip AE. Natural History and Therapeutic Management of Acute Aortic Syndrome. Rev Esp Cardiol [Internet]. 1 de julio de 2004 [citado 15 de marzo de 2019];57(07):667-79. Disponible en: http://www.revespcardiol.org/en/natural-history-andtherapeutic-management/articulo/13064218/

3. Patil TA, Nierich A. Transesophageal echocardiography evaluation of the thoracic aorta. Ann Card Anaesth [Internet]. octubre de 2016 [citado 9 de marzo de 2019];19(Suppl 1):S44-55. Disponible en: https://www.ncbi.nlm.nih.gov/pmc/articles/PMC5100242/ 
4. Asouhidou I, Asteri T. Acute aortic dissection: be aware of misdiagnosis. BMC Res Notes [Internet]. 20 de febrero de 2009 [citado 15 de marzo de 2019]; 2:25. Disponible en: https://www.ncbi.nlm.nih.gov/pmc/articles/PMC2653043/

5. Augoustides JG, Geirsson A, Szeto WY, Walsh EK, Cornelius B, Pochettino A, et al. Observational study of mortality risk stratification by ischemic presentation in patients with acute type A aortic dissection: the Penn classification. Nature Reviews Cardiology [Internet]. febrero de 2009 [citado 7 de marzo de 2019];6(2):140-6. Disponible en: https://www.nature.com/articles/ncpcardio1417

6. Petrilli G, Puppini G, Torre S, Calzaferri D, Bugana A, Faggian G. An Unusual Complication of Surgery for Type A Dissection Treated by Thoracic Endovascular Aortic Repair. Aorta (Stamford) [Internet]. 1 de julio de 2013 [citado 7 de marzo de 2019];1(2):131-4. Disponible en: https://www.ncbi.nlm.nih.gov/pmc/articles/PMC4682713/

7. Mokashi SA, Svensson LG. Guidelines for the management of thoracic aortic disease in 2017. Gen Thorac Cardiovasc Surg. enero de 2019;67(1):59-65.

8. Mehta RH, O'Gara PT, Bossone E, Nienaber CA, Myrmel T, Cooper JV, et al. Acute type A aortic dissection in the elderly: clinical characteristics, management, and outcomes in the current era. Journal of the American College of Cardiology [Internet]. 21 de agosto de 2002 [citado 11 de marzo de 2019];40(4):685-92. Disponible en: http://www.sciencedirect.com/science/article/pii/S0735109702020053

9. Fukui T. Management of acute aortic dissection and thoracic aortic rupture. J Intensive Care [Internet]. 1 de marzo de 2018 [citado 9 de marzo de 2019];6. Disponible en: https://www.ncbi.nlm.nih.gov/pmc/articles/PMC5831732/

10. Kim JS, Park K-H, Lim C, Kim DJ, Jung Y, Shin YC, et al. Prediction of Intimal Tear Site by Computed Tomography in Acute Aortic Dissection Type A. Korean Circ J [Internet]. enero de 2016 [citado 9 de marzo de 2019];46(1):48-55. Disponible en: https://www.ncbi.nlm.nih.gov/pmc/articles/PMC4720849/

11. Lu D, Li C-L, Lv W-F, Ni M, Deng K-X, Zhou C-Z, et al. Diagnostic value of multislice computerized tomography angiography for aortic dissection: A comparison with DSA. Exp Ther Med [Internet]. febrero de 2017 [citado 9 de marzo de 2019];13(2):405-12. Disponible en: https://www.ncbi.nlm.nih.gov/pmc/articles/PMC5348692/

12. Sobczyk D, Nycz K. Feasibility and accuracy of bedside transthoracic echocardiography in diagnosis of acute proximal aortic dissection. Cardiovasc Ultrasound [Internet]. 25 de marzo de 2015 [citado 9 de marzo de 2019];13. Disponible en: https://www.ncbi.nlm.nih.gov/pmc/articles/PMC4396118/

13. Wang H, Wagner M, Benrashid E, Keenan J, Wang A, Ranney D, et al. Outcomes of Reoperation After Acute Type A Aortic Dissection: Implications for Index Repair Strategy. J Am Heart Assoc [Internet]. 3 de octubre de 2017 [citado 7 de marzo de 2019];6(10). Disponible en: https://www.ncbi.nlm.nih.gov/pmc/articles/PMC5721847/ 


\section{PARA CITAR EL ARTÍCULO INDEXADO.}

Trelles Correa, M., Yamunaque Vire, H., \& Luna Castro, E. (2019). Disección Aórtica manejo clínico quirúrgico a propósito de 4 casos. Ciencia Digital, 3(4.2), 160-167. https://doi.org/10.33262/cienciadigital.v3i4.2.1020

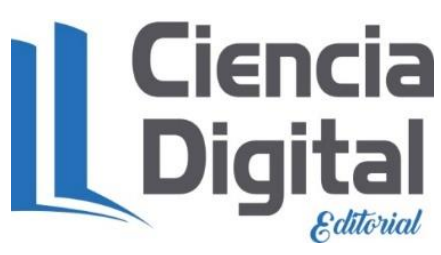

El artículo que se publica es de exclusiva responsabilidad de los autores y no necesariamente reflejan el pensamiento de la Revista Ciencia Digital.

El artículo queda en propiedad de la revista y, por tanto, su publicación parcial y/o total en otro medio tiene que ser autorizado por el director de la Revista Ciencia Digital.
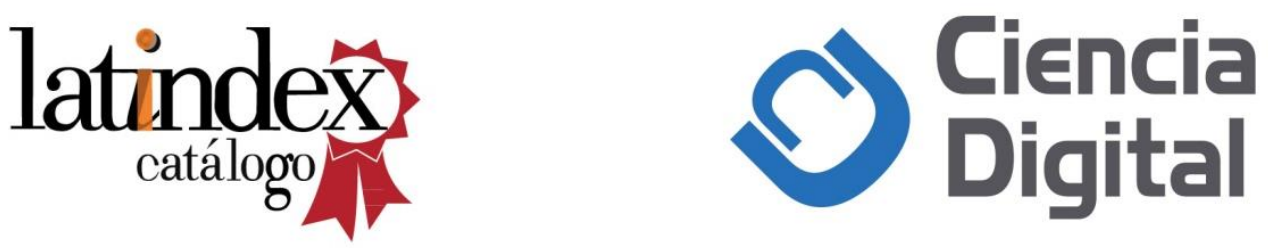